\title{
Atrial Remodeling in Pregnant Hypertensive Women: Comparison between Chronic and Gestational Hypertension
}

\author{
Anna Vittoria Mattioli ${ }^{*}, 1,2$, Sonia Pennella ${ }^{1,2}$, Fabrizio Demaria $^{3}$ and Alberto Farinetti ${ }^{4}$ \\ ${ }^{I}$ Department of Biomedical Science, University of Modena and Reggio Emilia, Modena, Italy \\ ${ }^{2}$ Istituto Nazionale di Ricerche Cardiovascolari, Bologna, Italy \\ ${ }^{3}$ Neonatal Intensive Care Unit, Ospedale Maggiore Bologna, Bologna, Italy \\ ${ }^{4}$ Department of Surgery, University of Modena and Reggio Emilia, Modena, Italy
}

\begin{abstract}
Objectives: Left atrial (LA) enlargement is a common finding in hypertensive patients (pts), however little information is available on LA changes during pregnancy. The present study evaluated LA size and function in pregnant women with hypertension.

Methods: Patients population included 30 women with chronic hypertension and 30 patients with gestational hypertension. A control group of 16 normotensive pregnant women was selected. Serial echocardiography was performed at 12 and 24 week of gestation in chronic hypertension and at 24 week in gestational hypertension and after delivery. LA diameters and volumes were measured and compared. LA conduit volume, passive and active emptying volumes were calculated. Patients were divided in 2 groups according to max LAV Index $>32 \mathrm{ml} / \mathrm{m}^{2}$ at 24 week of gestation.

Results: Patients with chronic hypertension showed higher LA diameters $(42 \pm 2.0$ vs. $36.5 \pm 1.8 ; \mathrm{p}<0.001)$ and LA volumes (maximal volume $45.5 \pm 4.0$ vs. $38.1 \pm 7.3 ; \mathrm{p}<0.001$ ) compared with patients with gestational hypertension. The median value of max LAVI was $32 \pm 6.2 \mathrm{ml} / \mathrm{m}^{2}$ in chronic and $26 \pm 5 \mathrm{ml} / \mathrm{m}^{2}$ in gestational hypertension $(\mathrm{p}<0.01)$. During follow-up patients with max LAVI $>32 \mathrm{ml} / \mathrm{m}^{2}$ had more clinical complications evaluated as increase of therapy, fluid retention that need diuretic and hospitalization.

Conclusion: Patients with chronic hypertension showed a more marked increased of LA volumes compared to gestational hypertension due to a remodeling of atrial shape as effect of chronic overload. Patients with more dilated LA volumes developed a greater number of clinical complications suggesting that a dilated LA could be a parameter of hemodynamic instability.
\end{abstract}

Keywords: Hypertension, left atrium, pregnancy, women.

\section{INTRODUCTION}

Chronic hypertension induces important functional and geometrical changes in left size of heart, involving both ventricle and atrium. The type and extent of remodeling depends on the strength and the duration of exposure to the "stressors" i.e. the duration of elevated blood pressure and the level of systolic pressure $[1,2]$. During diastole, the left atrium (LA) is directly exposed to pressures in left ventricle that increased with the decrease of left ventricular compliance, subsequently the LA pressure increase to maintain adequate filling and the rise in atrial wall tension leads to chamber dilatation and stretching of the atrial myocardium $[3,4]$.

In patients with hypertension left atrial enlargement may developed early. Mechanisms for accelerated LA remodeling included severe diastolic dysfunction, deranged plasma

*Address correspondence to this author at the Department of Biomedical Science, Cardiology, University of Modena and R.E., Via del pozzo, 71, 41100 Modena, Italy; Tel: 0039/59/4224043; Fax: 0039/59/4224323;

E-mail: annavittoria.mattioli@unimore.it volume control, intensified neurohormonal activation, as well as development of an atrial myopathy secondary to oxidative stress and lipoapoptosis [5-7].

Hemodynamic changes (i.e. increased plasma volume) occur early in pregnancy and persisted during all time of gestation $[8,9]$. In non-pregnant women, when volume increases of the magnitude seen in pregnancy it rapidly returned to baseline levels through humoral and neural mechanism [10]. The sensitivity of these regulatory mechanisms is significantly attenuated in pregnancy, such that the normal hypervolemia of pregnancy is maintained. The atrial distention is involved in these control mechanisms and it is possible that LA atrial changes induced by chronic hypertension influenced hemodynamic responses during pregnancy. Although the ventricular changes that occur during pregnancy have been the subject of intense study, the effect of pregnancy on left atrial size and shape has received little investigative attention.

The aim of the present study was to evaluated changes in LA geometry and function in pregnant women with chronic hypertension compared with women with gestational hypertension. 
Table 1. Baseline Characteristics of the Study Groups

\begin{tabular}{|c|c|c|c|c|}
\hline Parameters & Chronic & Gestational & Normotensive & $\mathbf{p}$ \\
\hline Mean Age (years) & $34 \pm 5$ & $33 \pm 6$ & $34 \pm 4$ & n.s. \\
\hline Height (m) & $1.64 \pm 0.1$ & $1.68 \pm 0.09$ & $1.66 \pm 0.2$ & n.s. \\
\hline Weight $(\mathrm{Kg})$ & $61 \pm 12$ & $65 \pm 11$ & $63 \pm 10$ & n.s. \\
\hline SBP (mmHg) & $144 \pm 16$ & $134 \pm 20$ & $112 \pm 12$ & $\begin{array}{c}0.05 \mathrm{Ch} \text { vs. Gh } \\
0.001 \mathrm{Nl} v s . \mathrm{Ch} \text { and } \mathrm{GH}\end{array}$ \\
\hline $\mathrm{DBP}(\mathrm{mmHg})$ & $90 \pm 10$ & $87 \pm 10$ & $80 \pm 5$ & $0.001 \mathrm{Nl} v s . \mathrm{Ch}$ and $\mathrm{GH}$ \\
\hline LVEF (\%) & $66 \pm 6$ & $67 \pm 9$ & $68 \pm 8$ & n.s. \\
\hline LVEDV (ml) & $110 \pm 15$ & $105 \pm 17$ & $96 \pm 17$ & $\begin{array}{c}0.001 \mathrm{Ch} v s . \mathrm{Gh} \\
0.001 \mathrm{Nl} v s . \mathrm{Ch} \text { and Gh }\end{array}$ \\
\hline LVESV (ml) & $36 \pm 10$ & $38 \pm 9$ & $25 \pm 8$ & $0.001 \mathrm{Nl} v s . \mathrm{Ch}$ and $\mathrm{Gh}$ \\
\hline $\operatorname{IVSd}(\mathrm{cm})$ & $1.12 \pm 0.09$ & $0.95 \pm 0.1$ & $0.77 \pm 0.2$ & $\begin{array}{c}0.001 \mathrm{Ch} v s . \mathrm{Gh} \\
0.001 \mathrm{Nl} v s . \mathrm{Ch} \text { and Gh}\end{array}$ \\
\hline PWd (cm) & $1.15 \pm 0.07$ & $0.97 \pm 0.09$ & $0.81 \pm 0.08$ & $\begin{array}{c}0.001 \mathrm{Ch} v s . \mathrm{Gh} \\
0.001 \mathrm{Nl} v s . \mathrm{Ch} \text { and Gh}\end{array}$ \\
\hline Mitral E/A & $0.85 \pm 0.02$ & $0.70 \pm 0.03$ & $1.10 \pm 0.05$ & $\begin{array}{c}0.001 \mathrm{Ch} v s . \mathrm{Gh} \\
0.001 \mathrm{Nl} v s . \mathrm{Ch} \text { and Gh }\end{array}$ \\
\hline MV DT (msec) & $230 \pm 15$ & $255 \pm 16$ & $172 \pm 29$ & $\begin{array}{c}0.001 \mathrm{Ch} v s . \mathrm{Gh} \\
0.001 \mathrm{Nl} v s . \mathrm{Ch} \text { and Gh }\end{array}$ \\
\hline $\mathrm{PV}$ S/D ratio & $1.1 \pm 0.2$ & $1.0 \pm 0.1$ & $1.0 \pm 0.1$ & n.s. \\
\hline TDI mitral E/E' & $11 \pm 3$ & $10 \pm 4$ & $10 \pm 1.3$ & n.s. \\
\hline
\end{tabular}

Abbreviations: $\mathrm{SBP}=$ systolic blood pressure; $\mathrm{DBP}=$ diastolic blood pressure; $\mathrm{MBP}=$ mean Blood Pressure; $\mathrm{LVEF}=$ left ventricular ejection fraction; $\mathrm{LVEDV}=$ left ventricle enddiastolic volume; LVESV= left ventricle end-diastolic volume; IVSD= Interventricular septum diastolic thickness; PWd= posterior wall diastolic thickness; MV DT mitral valve deceleration time; $\mathrm{PV} \mathrm{S} / \mathrm{D}=$ pulmonary vein systolic/diastolic ratio; $\mathrm{TDI}=$ tissue Doppler imaging; $\mathrm{n} . \mathrm{s} .=$ non significant; $\mathrm{CH}=$ chronic; $\mathrm{GH}=$ gestastional; $\mathrm{Nl}=$ normotensive

\section{METHODS}

\section{Study Population}

Study groups included 34 pregnant women with chronic hypertension (mean age $34 \pm 5$ years), 30 of whom were in their first pregnancy and 4 who had a previous uncomplicated pregnancy, and 35 pregnant women who developed gestational hypertension (mean age $33 \pm 6$ years), 30 during their first pregnancy and five who had a previous uncomplicated pregnancy.

A control group of normotensive pregnant women was included (Table 1).

Chronic and gestational hypertensions were defined according to the Report of the National High Blood Pressure Education Program Working Group on High Blood Pressure in Pregnancy [11]. Chronic hypertension was defined as hypertension that is present and observable before pregnancy or that is diagnosed before the 20th week of gestation. Hypertension was defined as a blood pressure $\geq 140 \mathrm{~mm} \mathrm{Hg}$ systolic or $\geq 90 \mathrm{~mm} \mathrm{Hg}$ diastolic. Hypertension that was diagnosed for the first time during pregnancy and that did not resolve post partum was also classified as chronic hypertension [11]. The mean duration of chronic hypertension was $29 \pm 10$ months.

Gestational blood pressure elevation was defined as a blood pressure $>140 \mathrm{~mm} \mathrm{Hg}$ systolic or $>90 \mathrm{~mm} \mathrm{Hg}$ diastolic in a woman who was normotensive before 20 weeks' gestation. In the absence of proteinuria the disease was suspected when increased blood pressure appeared accompanied by the following symptoms: headache, blurred vision, and abdominal pain, or by abnormal laboratory test results, specifically low platelet counts and abnormal liver enzyme values [11]. Blood pressure measurements were obtained by the average of multiple readings and for many patients measurement were taken even outside the clinician's office (e.g., home blood pressure monitoring or ambulatory blood pressure monitoring).

Patients with gestational hypertension were not taking any medication at the time of enrollment. During pregnancy all patients were treated with methyldopa $250-500 \mathrm{mg}$ /day. All patients were in sinus rhythm. Exclusion criteria were: diabetes, previous episodes of supraventricular arrhythmias, clinical symptoms or signs of heart failure, a global ejection fraction less than $50 \%$ on echocardiographic examinations, undetermined gestational age, tobacco use, poor echocardiographic windows not allowed echo measurements and preexisting chronic medical problems. We also excluded patients with a prior pregnancy complicated by severe preeclampsia or intrauterine growth restriction.

The protocol was approved by the Internal Ethics Committee and all patients signed an informed consent form.

\section{Doppler Echocardiography}

A complete mono- and two-dimensional color Doppler echocardiogram was performed on each patient using a commercial Hewlett Packard echocardiograph. 
LA function was assessed using the following parameters:

- Transmitral pulsed Doppler was recorded from the apical 4-chamber view, with the sample volume positioned between the tips of the mitral leaflets. Peak early filling (E) and atrial filling (A) velocities were recorded. Deceleration time and pressure half time was measured from the transmitral flow pattern.

The atrial ejection force (dynes) was evaluated using the following equation:

- $\quad 0.5 \times 1.06 \times$ mitral orifice area $\times$ (peak A velocity) ${ }^{2}$ $[12,13]$.

The following parameters were measured and compared:

- LA size was measured during systole along the parasternal long axis view from 2-dimensionallyguided M-mode tracing and from the apical four chamber view [14];

- $\quad$ LA volumes were measured from the apical 4-chamber and 2-chamber views by means of the biplane area-length method [15]. LA volumes $\left(\mathrm{cm}^{3} / \mathrm{m}^{2}\right)$ were determined just before the mitral valve opening (maximal volume, max LAV), at the onset of atrial systole ( $\mathrm{p}$ wave of electrocardiogram, $\mathrm{P}$ volume), and at mitral valve closure (minimal volume, min LAV) [16]. From the LA volumes the following parameters were estimated: LA passive emptying was assessed as: LA passive emptying volume (max LAV-P volume, $\left(\mathrm{cm}^{3} / \mathrm{m}^{2}\right)$ ), conduit volume [left ventricular stroke volume- (Max LAV-min LAV) $\left.\left(\mathrm{cm}^{3} / \mathrm{m}^{2}\right)\right]$ and LA passive emptying fraction (LA passive emptying volume/max LAV); LA active emptying was estimated using: LA active emptying volume ( $P$ volume-min LAV $\left(\mathrm{cm}^{3} / \mathrm{m}^{2}\right)$ ), and LA active emptying fraction (LA active emptying volume/P volume); LA total emptying volume was also calculated (max LAV-min LAV $\left(\mathrm{cm}^{3} / \mathrm{m}^{2}\right)$ ). LA emptying fraction (LAEF) was calculated via dividing the difference between max LAV and min LAV by max LAV. It was used as a parameter of the global LA contractile function.

The max LAV index (LAVI) was calculated as ratio to body surface area and LA enlargement [16]. Patients were divided in 2 groups according to LAVI $>32 \mathrm{ml} / \mathrm{m}^{2}$ at the time of echo control performed at 24 weeks of gestation.

Left Ventricular (LV) internal dimension, and septum and posterior wall thickness were measured according to the American Society of Echocardiography guidelines [14]. LV mean wall thickness was determined as the mean value of septum and posterior wall thickness at the end of diastole [17].

\section{Follow-Up}

Serial echocardiographic examinations were performed at 12 and 24 week of gestation and 1 month after delivery in women with chronic hypertension and at 24 week of gestation and 1 month after delivery in gestational hypertension. Normotensive patients were evaluated at 12 and 24 weeks of gestation and after delivery.

\section{Statistical Analysis}

Data are expressed as mean value $\pm 1 \mathrm{SD}$ for continuous variables. The statistical significance of serial changes in echocardiographic parameters was determined by 1-way analysis of variance for repeated measures. Comparison between groups was analyzed by independent $t$ test. A statistical software (SPSS Version 11.0 SPSS, Inc) was used for statistical analysis. A p value $<0.05$ (2-tailed) was considered statistically significant.

\section{RESULTS}

Clinical characteristics and echocardiographic data of patients are shown in Table 1. All patients with chronic hypertension had the diagnosis before the 12 week of gestation. In patients with chronic hypertension we com-pared changes developed during pregnancy (12 versus 24 week of

Table 2. Echo Parameters Related to Left Atrium: Comparison between Data Obtained at 12 and 24 Week of Gestation in Patients with Chronic Hypertension and after Delivery

\begin{tabular}{|c|c|c|c|c|c|}
\hline Parameters & 12 week & 24 week & $\mathbf{p}$ & After delivery & $\mathbf{p}$ \\
\hline LA long axis diam (mm) & $50.7 \pm 2.8$ & $53.5 \pm 2.1$ & 0.001 & $49.0 \pm 2.0$ & $0.001 v s .24 \mathrm{wk}$ \\
\hline Minimal vol $\left(\mathrm{cm}^{3} / \mathrm{m}^{2}\right)$ & $19.1 \pm 2.0$ & $21.0 \pm 3.2$ & n.s. & $20.0 \pm 2.6$ & n.s. \\
\hline $\mathrm{P}$ atrial vol $\left(\mathrm{cm}^{3} / \mathrm{m}^{2}\right)$ & $22.0 \pm 1.9$ & $20.2 \pm 2.1$ & 0.001 & $21.1 \pm 2.1$ & n.s. \\
\hline Passive empt vol $\left(\mathrm{cm}^{3} / \mathrm{m}^{2}\right)$ & $12.0 \pm 1.5$ & $15.2 \pm 1.1$ & 0.001 & $12.5 \pm 1.4$ & $0.001 v s .24 \mathrm{wk}$ \\
\hline Passive emptying fraction & $0.55 \pm 0.1$ & $0.81 \pm 0.2$ & 0.001 & $0.55 \pm 0.3$ & $0.001 v s .24 \mathrm{wk}$ \\
\hline Active empt vol $\left(\mathrm{cm}^{3} / \mathrm{m}^{2}\right)$ & $8.5 \pm 1.2$ & $7.2 \pm 2.0$ & n.s. & $8.1 \pm 2.1$ & $0.001 v s .24 \mathrm{wk}$ \\
\hline Active emptying fraction & $0.38 \pm 0.1$ & $0.34 \pm 0.1$ & n.s. & $0.39 \pm 0.2$ & 0.001 vs. $24 \mathrm{wk}$ \\
\hline LA total empt vol $\left(\mathrm{cm}^{3} / \mathrm{m}^{2}\right)$ & $21.5 \pm 2.5$ & $22.8 \pm 2.3$ & n.s. & $21.7 \pm 2.3$ & n.s. \\
\hline
\end{tabular}


gestation) and after delivery. Then we compared data obtained at 24 week of gestation and after delivery in patients with chronic and gestational hypertension and with normal pregnancy.

\section{Chronic Hypertension (Table 2)}

At baseline LA dimensions were greater than normal, and further increased at 24 week of gestation. Maximal volume and LA passive emptying volume significantly increased. On contrary $\mathrm{P}$ atrial volume significantly decreased. Minimal LA volume, LA active emptying volume and LA total emptying volume did not change significantly. Atrial function was evaluated by using atrial ejection force, a parameter of atrial systolic function, and LAEF. LAEF increased from $44 \pm 4$ to $46 \pm 3, p=0.008$ trend to significance while AEF didn't show significantly variation during pregnancy. After delivery the left atrium tended to reduce and volumes tended to return to baseline values.

\section{Comparison between Chronic and Gestational Hypertension (Table 3)}

The antero-posterior left atrial diameter and the long axis diameter, measured from 4 chamber view, were greater in chronic hypertension compared with gestational hypertension (respectively, $42.0 \pm 2.0$ vs. $36.5 \pm 1.8 ; \mathrm{p}<0.001$ and $53.5 \pm 2.1$ vs. $50.2 \pm 2.5 ; \mathrm{p}<0.001)$. Normotensive women had smaller diameters compared to chronic and gestational hypertension. The mean LA max and minimal volumes were higher in chronic hypertension compared to gestational hypertension while $\mathrm{P}$ atrial volume was similar in the 3 groups. Conduit volume was significantly smaller in chronic hypertension. LAEF was increased in chronic hypertension $(46 \pm 3 v s .36 \pm 4.4, \mathrm{p}=0.0001)$ while AEF was similar in all patients (Table $\mathbf{3}$ ).

In chronic and gestational hypertension the median LA $\max$ volume index was $32 \pm 6.2 \mathrm{ml} / \mathrm{m}^{2}$ and $26 \pm 5 \mathrm{ml} / \mathrm{m}^{2}$ respectively $(\mathrm{p}<0.01)$, whereas it was $24 \pm 3.3 \mathrm{ml} / \mathrm{m}^{2}$ $(\mathrm{p}<0.001 v s$. chronic and gestational $)$ in normotensive patients. In chronic hypertension we reported a LAVI $>32$ $\mathrm{ml} / \mathrm{m}^{2}$ in 17 patients, whereas in gestational hypertension it was found in 5 patients, none in normal patients. During follow-up patients with max LAVI greater than $32 \mathrm{ml} / \mathrm{m}^{2}$ had more clinical complications: 5 patients need an adjustment of the dose of antihypertensive therapy, 7 patients had fluid retention that needed diuretic therapy ( 4 of them were treated with furosemide and 3 patients were treated with hydralazine) and 11 patients needed hospitalization to control and treat blood pressure. The rate of preterm delivery was low: 3 patients delivered before 32 weeks and 2 other patients delivered between 32 and 34 weeks. After delivery these five patients maintained a dilated left atrium. In the group of patients with LAV index smaller than $32 \mathrm{ml} / \mathrm{m}^{2}$ complications occurred in 5 patients. Data were not subjected to statistical analysis because of the small number of events.

LV end-diastolic and LV end-systolic volumes slightly increased during pregnancy but changes were not statically significant. Ejection fraction was also preserved (data no show).

Table 3. Comparison between Patients with Chronic Hypertension and Patients with Gestational Hypertension at 24 Weeks of Gestation

\begin{tabular}{|c|c|c|c|c|c|}
\hline Parameters & Chronic (Ch) & Gestational (Gh) & $\mathbf{p}$ & Normal (NI) & $\mathbf{p}$ \\
\hline LA ant-post diam (mm) & $42.0 \pm 2.0$ & $36.5 \pm 1.8$ & 0.001 & $35.2 \pm 1.0$ & $\begin{array}{l}0.01 \text { vs. Ch } \\
\text { n.s. vs. Gh }\end{array}$ \\
\hline LA long axis diam (mm) & $53.5 \pm 2.1$ & $50.2 \pm 2.5$ & 0.001 & $45.2 \pm 1.7$ & $\begin{array}{l}0.001 \text { vs. Ch } \\
0.001 \text { vs. Gh }\end{array}$ \\
\hline Maximal vol $\left(\mathrm{cm}^{3} / \mathrm{m}^{2}\right)$ & $45.5 \pm 4.0$ & $38.1 \pm 7.3$ & 0.001 & $35.1 \pm 3.2$ & $\begin{array}{l}0.001 \text { vs. Ch } \\
0.001 \text { vs. Gh }\end{array}$ \\
\hline $\mathrm{P}$ atrial vol $\left(\mathrm{cm}^{3} / \mathrm{m}^{2}\right)$ & $20.2 \pm 2.1$ & $19.4 \pm 3.5$ & n.s. & $18.3 \pm 2.8$ & n.s. \\
\hline Conduit vol $\left(\mathrm{cm}^{3} / \mathrm{m}^{2}\right)$ & $33.4 \pm 3.3$ & $38.0 \pm 3.1$ & 0.001 & $27.3 \pm 4.5$ & $\begin{array}{l}0.001 \text { vs. Ch } \\
0.001 \text { vs. Gh }\end{array}$ \\
\hline Active empt vol $\left(\mathrm{cm}^{3} / \mathrm{m}^{2}\right)$ & $7.2 \pm 2.0$ & $9.7 \pm 1.8$ & 0.001 & $5.2 \pm 1.8$ & $\begin{array}{l}0.05 \text { vs. Ch. } \\
0.001 \text { vs. Gh }\end{array}$ \\
\hline Active emptying fraction & $0.34 \pm 0.1$ & $0.49 \pm 0.8$ & 0.001 & $0.41 \pm 0.1$ & $\begin{array}{l}0.001 \text { vs. Ch } \\
0.001 \text { vs. Gh }\end{array}$ \\
\hline LA total emptying vol $\left(\mathrm{cm}^{3} / \mathrm{m}^{2}\right)$ & $22.8 \pm 2.3$ & $19.6 \pm 2.2$ & 0.001 & $17.8 \pm 4.0$ & $\begin{array}{c}0.001 \text { vs. Ch } \\
\text { n.s. vs. Gh }\end{array}$ \\
\hline Atrial Ejection Force (dynes) & $13.5 \pm 2.4$ & $13.2 \pm 2.4$ & n.s. & $15.2 \pm 1.9$ & $\begin{array}{l}0.001 \text { vs. Ch } \\
0.001 \text { vs. Gh }\end{array}$ \\
\hline
\end{tabular}

Legend: see Table $\mathbf{1}$. 


\section{DISCUSSION}

The present study evaluated the effects of pregnancy on LA size and function in women with chronic and gestational hypertension.

The major finding was that patients with chronic hypertension had a more dilated LA compared to patients with gestational hypertension. We hypothetize that this difference could be the effect of a previous LA remodeling in chronic hypertension, however some changes occurring in geometry and function reversed after delivery in both groups of patients, suggesting a role of pregnancy related hemodynamic changes.

The behavior of pressure in normal pregnancy is well known; the diastolic pressure drops to an average of 10 $\mathrm{mmHg}$ below no pregnant values by mid-pregnancy, then slowly approaches no gravid levels in the third trimester. Women with chronic hypertension tend to have even greater decreases in their blood pressure in early pregnancy, and their "normal" rise in the $3^{\text {rd }}$ trimester may be misdiagnosed as preeclampsia [18].

A different patho-physiological mechanism seems to be involved in gestational hypertension, in which a spasm of small arteries all over the body causes an increase in peripheral vascular resistance $[19,20]$. The factors causing the spasm of the small arteries might have a similar effect on the myocardium. This lead to a different hemodynamic balance in chronic and gestational hypertension, however, due to the complex cluster of mechanisms involved in hypertension, we cannot exclude a contribution of generalized small arteries spasm in chronic hypertension leading to increased arterial resistance. Recently, a paper from Powe and coworkers has identified an imbalance of proangiogenic and antiangiogenic proteins as a key factor in the pathogenesis of the preeclampsia [21].

Several studies found that chronic hypertension induced functional and anatomical remodeling in left atrium $[1,2,7]$. LA reservoir and pump function are increased in hypertensive patients with impaired LV relaxation. During ventricular systole, the LA reservoir volume compensated the impaired early LV filling [7].

We found that in chronic hypertension, LA diameters and LA maximal volume increased progressively from $12^{\text {th }}$ to $24^{\text {th }}$ week of gestation. Similarly the passive emptying volumes contribution to LV stroke volume increased whereas LA active emptying volume and conduit volume remain unchanged. These results were in agreement with previous study that found a direct correlation between max LAV and LA passive emptying volume in normal subjects [22]. During pregnancy, the active emptying fraction reduced, suggesting a reduction in the LA reservoir function. LA reservoir function is related to LA relaxation and compliance as well as LV longitudinal systolic fiber shortening $[23,24]$. Thus, reduced LA reservoir function reflected not only the extent of atrial remodeling but also the underlying ventricular dysfunction which contributed to LA remodeling. LA phasic dysfunction, specifically the impairment of LA reservoir function, represented a more advanced phase of LA remodeling developing after LA enlargement [25].
After delivery, the reduction of LA passive emptying volume and fraction suggested a normalization of LA function. Patients with chronic hypertension had a LA remodeling secondary to chronic remodeling of the left ventricle and to an enhancement of left atrial changes developed before pregnancy. Further changes in atrial function depended upon volume overload during pregnancy and were reversible.

In patients with gestational hypertension changes of LA dimension and volumes were similar to that observed in chronic hypertension, although women with chronic hypertension had greater LA size at baseline as the effect of longlasting pressure loading.

LA diameters and volumes were increase in women with gestational hypertension compared to normotensive women; however they were smaller than that observed in women affected by chronic hypertension. The conduit volume was more increased in gestational hypertension compared to chronic hypertension, suggesting that early diastolic filling was acutely augmented in these patients, whereas the compensatory late filling mechanism was reduced in gestational hypertension. In gestational hypertension the left atrium was previously normal and the pressure overload was brief, this short-lasting overload induced a temporarily increase in left ventricular volumes and an impairment of diastolic dysfunction. Similarly, the atrial dysfunction reported in patients affected by gestational hypertension was transient and the atrial shape changes were similar to that observed in normotensive pregnant women.

After delivery the LA of women with gestational hypertension was reduced suggesting that changes developing during pregnancy were reversible and were mighty induced by the fluid overload.

Complications were more frequent in women having LA volume index values $>32 \mathrm{ml} / \mathrm{m}^{2}$, both with chronic and gestational hypertension. We use this cut-off because Moller and coworkers found that a LA volume index $>32 \mathrm{ml} / \mathrm{m}^{2}$ was a powerful predictor of mortality after acute myocardial infarction and provided prognostic information incremental to clinical data [26].

Due to a dilated LA at baseline the number of women with chronic hypertension was higher. These preliminary observations, on a small number of subjects, suggested that a dilated LA could a marker of a provisional imbalance and that these patients would benefit from more frequent followup visit to avoid complications.

\section{CONCLUSION}

Changes in left atrial size and function are common in patients affected by chronic and gestational hypertension. Atrial function is reduced in patients with chronic hypertension compared to normotensive and gestational hypertension as effect of long-lasting pressure overload. Despite the limitation related to the low number of patients that developed complications we believe that the present study provides important insight in term of pathophysiology of hypertension during pregnancy and the relation between left atrial changes and complications. Due to the limited number of adverse events we reported, we suggest that further studies are needed to specifically address this issue. 


\section{CONFLICT OF INTEREST}

No conflict of interest

\section{ACKNOWLEDGEMENT}

None declared.

\section{REFERENCES}

[1] Gerdts E, Oikarinen L, Palmieri V, et al. Correlates of left atrial size in hypertensive patients with left ventricular hypertrophy. The losartan intervention for endpoint reduction in hypertension (LIFE) study. Hypertension 2002; 39: 739-43.

[2] Vaziri SM, Larson MG, Lauer MS, Benjamin EJ, Levy D. Influence of blood pressure on left atrial size: the framingham heart study. Hypertension 1995; 25: 1155-60.

[3] Greenberg BH, Chatterjee K, Parmley W, Werner J, Holly A. The influence of left ventricular filling pressure on atrial contribution to cardiac output. Am Heart J 1979; 98: 742-51.

[4] Tsang TSM, Barnes ME, Gersh BJ, Bailey KR, Seward JB. Left atrial volume as a morphologic expression of left ventricular diastolic dysfunction and relation to cardiovascular risk burden. Am J Cardiol 2002; 90: 1284-9.

[5] Casaclang-Verzosa G, Gersh BJ, Tsang TSM. Structural and functional remodeling of the left atrium clinical and therapeutic implications for atrial fibrillation. J Am Coll Cardiol 2008; 51: 111.

[6] Galderisi M, Petrocelli A, Fakher A, Izzo A, Alfieri A, de Divitiis $\mathrm{O}$. Influence of nighttime blood pressure on left atrial size in uncomplicated arterial systemic hypertension. Am J Hypertens 1997; 10: 836-42.

[7] Mattioli AV, Bonatti S, Mattioli G. Left atrial anatomy and function after atrial fibrillation of brief duration in hypertrophic hearts. Angiology 2006: 57: 7171-3.

[8] Brown MA, Zammit VC, Mitar DM. Extracellular fluid volumes in pregnancy-induced hypertension. J Hypertens 1992; 10: 61-8.

[9] Hays PM, Cruikshank DP, Dunn LJ. Plasma volume determinations in normal and preeclamptic pregnancies. Am J Obstet Gynecol 1985; 151: 958-66.

[10] Deng Y, Kaufman S. Effect of pregnancy on activation of central pathways following atrial distension. Am J Physiol Regul Integr Comp Physiol 1995; 269: R552-6.

[11] Report of the National High Blood Pressure Education Program Working Group on High Blood Pressure in Pregnancy. Am J Obstet Gynecol 2000; 183: S1-S23.
[12] Manning WJ, Silverman M, Katz SE, Douglas PS. Atrial ejection force: a noninvasive assessment of atrial systolic function. J Am Coll Cardiol 1993; 22: 221-5.

[13] Mattioli AV, Castelli A, Bastia E, Mattioli G. Atrial ejection force in patients with atrial fibrillation: comparison between DC shock and pharmacological cardioversion. Pacing Clin Electrophysiol 1999; $22: 33-8$

[14] Sahn DJ, DeMaria A, Kisslo J, Weiman A. Recommendations regarding quantitation in M-mode echocardiography: results of a survey of echocardiographic measurements. Circulation 1978; 58: 1072-83.

[15] Mattioli AV, Sansoni S, Ricci Lucchi G, Mattioli G. Serial evaluation of left atrial dimension after cardioversion for atrial fibrillation and relation to atrial function. Am J Cardiol 2000; 85: 832-6.

[16] Pritchett AM, Jacobsen SJ, Mahoney DW, Rodeheffer RJ, Bailey $\mathrm{KR}$, Redfield MM. Left atrial volume as an index of left atrial size: a population-based study. J Am Coll Cardiol 2003; 41: 1036-43.

[17] Levy D, Savage DD, Garrison RJ, Anderson KM, Kannel WB, Castelli WP. Echocardiographic criteria for left ventricular hypertrophy: the framingham heart Study. Am J Cardiol 1987; 59: 956-60.

[18] Peters RM, Flack JM. Hypertensive disorders of pregnancy. J Obstet Gynecol Neonatal Nurs 2004; 33: 209-20.

[19] Visser W, Wallenburg HC. Central hemodynamic observations in untreated preeclamptic patients. Hypertension 1991; 17: 1072-7.

[20] Yuan L, Duan Y, Cao T. Echocardiographic study of cardiac morphological and functional changes before and after parturition in pregnancy-induced hypertension. Echocardiography 2006; 23 177-82.

[21] Powe CE, Levine RJ, Karumanchi SA. Preeclampsia, a disease of the maternal endothelium: the role of antiangiogenic factors and implications for later cardiovascular disease. Circulation 2011; 123: 2856-69.

[22] Williams JF, Sonnenblick EH, Braunwald E. Determinants of atrial contractile force in the intact heart. Am J Physiol 1965; 209: 10618.

[23] Barbier P, Solomon SB, Schiller NB, Glantz SA. Left atrial relaxation and left ventricular systolic function determine left atrial reservoir function. Circulation 1999; 100: 427-36.

[24] Suga H. Importance of atrial compliance in cardiac performance. Circ Res 1974; 35: 39-43.

[25] Abhayaratna WP, Fatema K, Barnes ME, et al. Left atrial reservoir function as a potent marker for first atrial fibrillation or flutter in persons $>65$ years of age. Am J Cardiol 2008; 101: 1626-9.

[26] Moller JE, Hillis JS, Oh JK, et al. Left atrial volume. A powerful predictor of survival after acute myocardial infarction. Circulation 2003: 107; 2207-12. 